Article

\title{
Certain Subclasses of Analytic Multivalent Functions Associated with Petal-Shape Domain
}

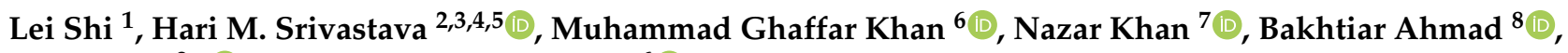 \\ Bilal Khan ${ }^{9, *}$ and Wali Khan Mashwani ${ }^{6}$ (D) \\ 1 School of Mathematics and Statistics, Anyang Normal University, Anyang 455002, China; \\ shimath@aynu.edu.cn \\ 2 Department of Mathematics and Statistics, University of Victoria, Victoria, BC V8W 3R4, Canada; \\ harimsri@math.uvic.ca \\ 3 Department of Medical Research, China Medical University Hospital, China Medical University, \\ Taichung 40402, Taiwan \\ 4 Department of Mathematics and Informatics, Azerbaijan University, 71 Jeyhun Hajibeyli Street, \\ AZ1007 Baku, Azerbaijan \\ 5 Section of Mathematics, International Telematic University Uninettuno, I-00186 Rome, Italy \\ 6 Institute of Numerical Sciences, Kohat University of Science and Technology, Kohat 26000, Pakistan; \\ ghaffarkhan020@gmail.com (M.G.K.); mashwanigr8@gmail.com (W.K.M.) \\ 7 Department of Mathematics, Abbottabad University of Science and Technology, Abbottabad 22010, Pakistan; \\ nazarmaths@gmail.com \\ 8 Govt. Degree College Mardan, Mardan 23200, Pakistan; pirbakhtiarbacha@gmail.com \\ 9 School of Mathematical Sciences and Shanghai Key Laboratory of PMMP, East China Normal University, \\ 500 Dongchuan Road, Shanghai 200241, China \\ check for \\ * Correspondence: bilalmaths789@gmail.com
}

updates

Citation: Shi, L.; Srivastava, H.M.; Khan, M.G.; Khan, N.; Ahmad, B.; Khan, B.; Mashwani, W.K. Certain Subclasses of Analytic Multivalent Functions Associated with Petal-Shape Domain. Axioms 2021, 10, 291. https://doi.org/10.3390/ axioms10040291

Academic Editor: Andriy Bandura

Received: 27 September 2021

Accepted: 31 October 2021

Published: 3 November 2021

Publisher's Note: MDPI stays neutral with regard to jurisdictional claims in published maps and institutional affiliations.

Copyright: (c) 2021 by the authors. Licensee MDPI, Basel, Switzerland. This article is an open access article distributed under the terms and conditions of the Creative Commons Attribution (CC BY) license (https:/ / creativecommons.org/licenses/by/ $4.0 /)$.

\begin{abstract}
In this article, we introduce a new class of multivalent analytic functions associated with petal-shape region. Furthermore, some useful properties, such as the Fekete-Szegö inequality, and their consequences for some special cases are discussed. For some specific value of function $f$, we obtain sufficient conditions for multivalent starlike functions connected with petal-shape domain. Finally, in the concluding section, we draw the attention of the interested readers toward the prospect of studying the basic or quantum (or $q$-) generalizations of the results, which are presented in this paper. However, the $(\mathfrak{p}, q)$-variations of the suggested $q$-results will provide a relatively minor and inconsequential development because the additional (rather forced-in) parameter $\mathfrak{p}$ is obviously redundant.
\end{abstract}

Keywords: multivalent functions; starlike functions; petal-shape domain; differential subordination; Fekete-Szegö inequality

\section{Introduction and Motivation}

To understand our main results and the notations used in this paper in a better way, some basic literature of Geometric Function Theory is presented here. For this understanding, we start with the class $\mathcal{A}$, the class of all regular or analytic functions $f$ in the open unit disk

$$
\mathbb{D}=\{z: z \in \mathbb{C} \text { and }|z|<1\}
$$

and satisfying the conditions

$$
f(0)=0 \quad \text { and } \quad f^{\prime}(0)=1 .
$$


Further, the class $\mathcal{S}$ represents all regular univalent functions $f$ of class $\mathcal{A}$. Let $\mathbb{B}_{0}$ be the class of Schwarz regular functions $w$ such that $w: \mathbb{D} \rightarrow \mathbb{D}$ with property that $w(0)=0$ and has power series expansion

$$
w(z)=\sum_{n=1}^{\infty} c_{n} z^{n}
$$

Furthermore, we briefly discuss the notion of subordinations; let $\Lambda_{1}, \Lambda_{2} \in \mathcal{A}$, then $\Lambda_{1}$, is said to subordinate to $\Lambda_{2}$ symbolically:

$$
\Lambda_{1} \prec \Lambda_{2}
$$

if there exists an analytic function $w(z)$ with properties that

$$
w(0)=0 \quad \text { and }|w(z)|<1,
$$

such that

$$
\Lambda_{1}(z)=\Lambda_{2}(w(z)) .
$$

Utilizing the idea of subordinations, different subclasses of starlike functions were introduced by Ma and Minda [1], in which the quantity $z f^{\prime}(z) / f(z)$ is subordinated to a more general maximal function $\Phi$ such that

$$
\frac{z f^{\prime}(z)}{f(z)} \prec \Phi(z)=\frac{1+z}{1-z} .
$$

In some particular cases, if we put $\Phi(z)=\frac{1+L z}{1+M z}$, then such functions are known as Janowski functions with condition on $L$ and $M$ is $-1 \leq M<L \leq 1$. These functions map the open unit disk to the disk in the right half plane with center on real axis and the end points of diameter are $\frac{1+L}{1+M}$ and $\frac{1-L}{1-M}$ (see [2]). If we vary $\Phi(z)$ to $1+\frac{4}{3} z+\frac{2}{3} z^{2}$, then the image of $\Phi(z)$ under open unit disk is bounded by cardioid; this case was studied by Sharma et al. [3]. Cho et al. [4] extended the idea to trigonometric function by replacing $\Phi(z)$ by $1+\sin (z)$. Furthermore, we put $\Phi(z)=2 / 1+e^{-z}$; this class was introduced by Goel and Kumar [5], in which they connected starlike functions to modified sigmoid functions. For more details of the above-mentioned interesting topics, we refer the reader to [6-9]. Recently, Ali et al. [10] obtained some conditions on $\alpha$, where $\alpha$ is any real number, such that

$$
1+\alpha \frac{z g^{\prime}(z)}{g^{i}(z)} \prec \sqrt{1+z} \Rightarrow g(z) \prec \sqrt{1+z} \quad(i=0,1,2,),
$$

where $g$ is a regular function defined on $\mathbb{D}$ with $g(0)=1$. Similar findings were carried out by various authors such as Kumar et al. [7,11,12], Paprocki and Sokół [13], Ahmad et al. [14], and Sharma et al. [15].

Let $\mathcal{A}_{p}$ denote the family of all regular $p$-valent functions $f$ in the open disk $\mathbb{D}$ having series representation

$$
f(z)=z^{p}+\sum_{n=p+1}^{\infty} a_{n} z^{n} \quad(z \in \mathbb{D}) .
$$

In recent years, many authors have studied the subclasses of multivalent ( $p$-valent) functions from different viewpoints and perspectives. For example, recently, Khan et al. [16,17] were essentially motivated by the Srivastava's published review article [18] and defined certain new families of multivalent $q$-starlike functions and studied some of their entrusting properties, such as inclusion results, radius problems, and sufficient conditions. Furthermore, Rehman et al. [19] defined and studied generalized subclasses of multivalent starlike functions. Recently, many well-known mathematicians have obtained the FeketeSzegö functional for different subclasses of analytic and bi-univalent functions, see for example [20-24]. 
Motivated by the above-mentioned work, we now introduce a new class $\mathcal{S}_{p}^{*}(p e t)$ of analytic multivalent starlike functions associated with the petal-shape domain.

Definition 1. A function $f \in \mathcal{A}_{p}$ will be in the class $\mathcal{S}_{p}^{*}(p e t)$ if it satisfies

$$
\frac{z f^{\prime}(z)}{p f(z)} \prec 1+\sinh ^{-1}(z) .
$$

The function $1+\sinh ^{-1}(z)$ is a multivalued function and has branch cuts along the line segments $(-\infty,-i) \cup(i, \infty)$ on the imaginary axis; hence, it is analytic in $\mathbb{D}$ and also the function $1+\sinh ^{-1}(z)$ maps the open unit disk onto petal-shape region, for example,

$$
\{w \in \mathbb{C}:|\sinh (w-1)|<1\} .
$$

Remark 1. If we take $p=1$, we have the class of analytic starlike functions associated with petal-shape domain introduced and studied by Kumar and Arora [25].

In this article, we first introduced a new class of multivalent analytic functions associated with petal-shape region. Some useful properties have been discussed, such as Fekete-Szegö inequality and their consequences to some special cases, and also evaluating conditions on $\alpha$ so that the following holds

$$
1+\frac{\alpha}{p} \frac{z^{1+p(i-1)} g^{\prime}(z)}{g^{i}(z)} \prec \frac{1+L z}{1+M z} \text {, for each } i=0,1,2,3
$$

then, $\frac{g(z)}{z^{p}}$ is proved to be subordinated to $1+\sinh ^{-1}(z)$. For some specific value of function $g$, we obtain sufficient conditions for multivalent starlike functions connected with the petal-shape domain.

\section{A Set of Lemmas}

To obtain our results, we need the following important Lemmas.

Lemma 1. [26] Let $w \in \mathbb{B}_{0}$ of the form (2), then for all $\lambda \in \mathbb{C}$, we have

$$
\left|c_{2}-\lambda c_{1}^{2}\right| \leq \max \{1,|\lambda|\}
$$

Lemma 2. [27] Let $w$ be a nonconstant regular function in $\mathbb{D}$ with $w(0)=0$; if

$$
\left|w\left(z_{0}\right)\right|=\max \left\{|w(z)|,|z| \leq\left|z_{0}\right|\right\}, z \in \mathbb{D}
$$

then, there exists a real number $m(m \geq 1)$ such that

$$
z_{0} w^{\prime}\left(z_{0}\right)=m w\left(z_{0}\right)
$$

\section{Main Results}

In this section, we start with the Fekete-Szegö problem for this newly defined class and their consequences to some special cases. Throughout our discussion, we assume that

$$
-1 \leq M<L \leq 1 \text { and } \alpha \in \mathbb{R} .
$$

Theorem 1. Let the function $f$ be of the form (3) and $f \in \mathcal{S}_{p}^{*}($ pet $)$, then,

$$
\left|a_{p+2}-\lambda a_{p+1}^{2}\right| \leq \frac{p}{2} \max \{1,|2 \lambda-1|\}
$$


and

$$
\left|a_{p+2}\right| \leq \frac{p}{2}
$$

Proof. For $f$ to be in the class $\mathcal{S}_{p}^{*}(p e t)$, there exists a Schwarz function $\varphi(z)$ such that

$$
\frac{z f^{\prime}(z)}{p f(z)}=1+\sinh ^{-1}(\varphi(z)) \text {. }
$$

Now,

$$
\frac{z f^{\prime}(z)}{p f(z)}=1+\frac{1}{p} a_{p+1} z+\left(\frac{2}{p} a_{p+2}-\frac{1}{p} a_{p+1}^{2}\right) z^{2}+\cdots
$$

and

$$
1+\sinh ^{-1}(\varphi(z))=1+c_{1} z+c_{2} z^{2}+\cdots
$$

From (7) and (8), we have

$$
\begin{gathered}
a_{p+1}=p c_{1} \\
a_{p+2}=\frac{p}{2} c_{2}+\frac{p^{2}}{2} c_{1}^{2} .
\end{gathered}
$$

From (9) and (10), we have

$$
\left|a_{p+2}-\lambda a_{p+1}^{2}\right|=\frac{p}{2}\left|c_{2}-(2 \lambda-1) c_{1}^{2}\right|
$$

using Lemma 1, we obtain the desired result (5). To prove (6), put $\lambda=0$ in (5) - the desired result is achieved.

Corollary 1. Let $f$ of the form (3) be in the class $\mathcal{S}_{p}^{*}($ pet $)$. Then,

$$
\left|a_{p+2}-a_{p+1}^{2}\right| \leq \frac{p}{2}
$$

Corollary 2. Let $f \in \mathcal{A}$ be of the form (3) belongs to the class $\mathcal{S}^{*}(p e t)$. Then,

$$
\left|a_{3}-\lambda a_{2}^{2}\right| \leq \frac{1}{2} \max \{1,|2 \lambda-1|\}
$$

and

$$
\begin{gathered}
\left|a_{3}\right| \leq \frac{1}{2} \\
\left|a_{3}-a_{2}^{2}\right| \leq \frac{1}{2} .
\end{gathered}
$$

Theorem 2. Let $g \in A_{p}$ and be of the form (3) satisfying the condition

$$
1+\frac{\alpha z^{1-p} g^{\prime}(z)}{p} \prec \frac{1+L z}{1+M z}
$$

with the restriction on $\alpha$ being

$$
|\alpha| \geq \frac{2^{\frac{1}{2}} p(L-M)}{1-|M|-2^{\frac{1}{2}} p\left(1+\sinh ^{-1}(1)\right)(1+|M|)}
$$

then,

$$
\frac{g(z)}{z^{p}} \prec 1+\sinh ^{-1}(z) .
$$


Proof. Let us define a function

$$
h(z)=1+\frac{\alpha z^{1-p} g^{\prime}(z)}{p},
$$

where $h(z)$ is analytic in $\mathbb{D}$ and $h(0)=1$. Further, consider

$$
\frac{g(z)}{z^{p}}=1+\sinh ^{-1}(w(z)) .
$$

To prove our result, we are required to show that $|w(z)|<1$. For this, consider

$$
h(z)=1+\alpha\left(1+\sinh ^{-1}(w(z))\right)+\frac{\alpha z w^{\prime}(z)}{p \sqrt{(w(z))^{2}+1}}
$$

and

$$
\begin{array}{r}
\left|\frac{h(z)-1}{L-M h(z)}\right|=\left|\frac{\alpha\left(1+\sinh ^{-1}(w(z))\right)+\frac{\alpha z w^{\prime}(z)}{p \sqrt{(w(z))^{2}+1}}}{L-M\left(1+\alpha\left(1+\sinh ^{-1}(w(z))\right)+\frac{\alpha z w^{\prime}(z)}{p \sqrt{(w(z))^{2}+1}}\right)}\right| \\
=\left|\frac{\alpha\left(1+\sinh ^{-1}(w(z))\right) p \sqrt{(w(z))^{2}+1}+\alpha z w^{\prime}(z)}{(L-M) p \sqrt{(w(z))^{2}+1}-M Y_{1}(p, \alpha, z)}\right|,
\end{array}
$$

where

$$
Y_{1}(p, \alpha, z)=\left(p \alpha \sqrt{(w(z))^{2}+1}\left(1+\sinh ^{-1}(w(z))\right)+\alpha z w^{\prime}(z)\right) .
$$

Now, suppose that for a point $z_{0} \in \mathbb{D}$ we have

$$
\max _{|z| \leq\left|z_{0}\right|}|w(z)|=\left|w\left(z_{0}\right)\right|=1 .
$$

Further, by Lemma 2, a number $m \geq 1$ exists with

$$
z_{0} w^{\prime}\left(z_{0}\right)=m w\left(z_{0}\right)
$$

In addition, we also suppose that

$$
w\left(z_{0}\right)=e^{i \theta} \quad(\theta \in[-\pi, \pi]) .
$$

Then, we have

$$
\begin{aligned}
\left|\frac{h\left(z_{0}\right)-1}{L-M h\left(z_{0}\right)}\right|= & \left|\frac{\alpha\left(1+\sinh ^{-1}\left(w\left(z_{0}\right)\right)\right) p \sqrt{\left(w\left(z_{0}\right)\right)^{2}+1}+\alpha z w^{\prime}\left(z_{0}\right)}{(L-M) p \sqrt{\left(w\left(z_{0}\right)\right)^{2}+1}-M Y_{2}\left(p, \alpha, z_{0}\right)}\right| \\
& \geq \frac{|\alpha| m-|\alpha|\left(1+\sinh ^{-1}\left(\left|e^{i \theta}\right|\right)\right) p \sqrt{e^{2 i \theta}+1}}{(L-M) p \sqrt{e^{2 i \theta}+1}+|M|\left(p|\alpha|\left(1+\sinh ^{-1}\left(\left|e^{i \theta}\right|\right)\right) \sqrt{e^{2 i \theta}+1}+|\alpha| m\right)} \\
& \geq \frac{|\alpha|\left(m-2^{\frac{1}{2}} p\left(1+\sinh ^{-1}(1)\right)\right)}{2^{\frac{1}{2}}(L-M) p+|\alpha||M|\left[m+2^{\frac{1}{2}} p\left(1+\sinh ^{-1}(1)\right)\right]}
\end{aligned}
$$

where

$$
\mathrm{Y}_{2}\left(p, \alpha, z_{0}\right)=\left(p \alpha \sqrt{\left(w\left(z_{0}\right)\right)^{2}+1}\left(1+\sinh ^{-1}\left(w\left(z_{0}\right)\right)\right)+\alpha z w^{\prime}\left(z_{0}\right)\right)
$$


Now, let

$$
k(m)=\frac{|\alpha|\left(m-2^{\frac{1}{2}} p\left(1+\sinh ^{-1}(1)\right)\right)}{2^{\frac{1}{2}}(L-M) p+|\alpha||M|\left[m+2^{\frac{1}{2}} p\left(1+\sinh ^{-1}(1)\right)\right]},
$$

then,

$$
k^{\prime}(m)=\frac{2^{\frac{1}{2}}|\alpha|(L-M) p+|M||\alpha|^{2} 2^{\frac{3}{2}} p\left(1+\sinh ^{-1}(1)\right)}{\left(2^{\frac{1}{2}}(L-M) p+|\alpha||M|\left[m+2^{\frac{1}{2}} p\left(1+\sinh ^{-1}(1)\right)\right]\right)^{2}}>0 .
$$

Clearly, $k(m)$ is an increasing function; so,

$$
\max k(m) \geq k(1) \quad(m \geq 1),
$$

and

$$
\left|\frac{h\left(z_{0}\right)-1}{L-M h\left(z_{0}\right)}\right| \geq \frac{|\alpha|\left(1-2^{\frac{1}{2}} p\left(1+\sinh ^{-1}(1)\right)\right)}{2^{\frac{1}{2}}(L-M) p+|M||\alpha|\left[1+2^{\frac{1}{2}} p\left(1+\sinh ^{-1}(1)\right)\right]} .
$$

From (13), we have

$$
\left|\frac{h\left(z_{0}\right)-1}{L-M h\left(z_{0}\right)}\right| \geq 1
$$

which is contradictory to the fact that

$$
h(z) \prec \frac{1+L z}{1+M z} .
$$

Thus, $|w(z)|<1$, and so, we obtain our desired result.

If we take $g(z)=\frac{z^{p+1} f^{\prime}(z)}{p f(z)}$ in Theorem 2, we have the following Corollary.

Corollary 3. If $f$ is in the class $\mathcal{A}_{p}$, has the form (3), and satisfies the condition

$$
1+\frac{\alpha z f^{\prime}(z)}{p^{2} f(z)}\left(p+1+\frac{z f^{\prime \prime}(z)}{f^{\prime}(z)}-\frac{z f^{\prime}(z)}{f(z)}\right) \prec \frac{1+L z}{1+M z},
$$

where the limitation on $\alpha$ is

$$
|\alpha| \geq \frac{2^{\frac{1}{2}} p(L-M)}{1-|M|-2^{\frac{1}{2}} p\left(1+\sinh ^{-1}(1)\right)(1+|M|)},
$$

then, $f \in \mathcal{S}_{p}^{*}($ pet $)$.

Proof. The proof is straightforward so it is left for the reader.

Theorem 3. Let $g \in A_{p}$ and be of the form (3), satisfying the condition

$$
1+\frac{\alpha}{p} \frac{z g^{\prime}(z)}{g(z)} \prec \frac{1+L z}{1+M z}
$$

where the limitation on $\alpha$ is

$$
|\alpha| \geq \frac{2^{\frac{1}{2}} p(L-M)\left(1+\sinh ^{-1}(1)\right)}{1-|M|-2^{\frac{1}{2}} p\left(1+\sinh ^{-1}(1)\right)(1+|M|)}
$$


then,

$$
\frac{g(z)}{z^{p}} \prec 1+\sinh ^{-1}(z)
$$

Proof. Let us define a function

$$
h(z)=1+\frac{\alpha}{p} \frac{z g^{\prime}(z)}{g(z)}
$$

where $h(z)$ is analytic in $\mathbb{D}$ and $h(0)=1$. Moreover, consider

$$
\frac{g(z)}{z^{p}}=1+\sinh ^{-1}(w(z)) .
$$

To show our result, we are required to show that $|w(z)|<1$. Now,

$$
h(z)=1+\alpha+\frac{\alpha z w^{\prime}(z)}{p\left(1+\sinh ^{-1}(w(z))\right) \sqrt{(w(z))^{2}+1}},
$$

and

$$
\begin{aligned}
& \left|\frac{h(z)-1}{L-M h(z)}\right|=\left|\frac{\alpha+\frac{\alpha z w^{\prime}(z)}{p\left(1+\sinh ^{-1}(w(z))\right) \sqrt{(w(z))^{2}+1}}}{L-M\left(1+\alpha+\frac{\alpha z w^{\prime}(z)}{p\left(1+\sinh ^{-1}(w(z))\right) \sqrt{(w(z))^{2}+1}}\right)}\right| \\
& \quad=\left|\frac{\alpha p\left(1+\sinh ^{-1}(w(z))\right) \sqrt{(w(z))^{2}+1}+\alpha z w^{\prime}(z)}{(L-M) p\left(1+\sinh ^{-1}(w(z))\right) \sqrt{(w(z))^{2}+1}-M Y_{3}(p, \alpha, z)}\right|
\end{aligned}
$$

where

$$
\mathrm{Y}_{3}(p, \alpha, z)=\left(\alpha p\left(1+\sinh ^{-1}(w(z))\right) \sqrt{(w(z))^{2}+1}+\alpha z w^{\prime}(z)\right)
$$

and suppose that any point $z_{0} \in \mathbb{D}$ such that

$$
\max _{|z| \leq\left|z_{0}\right|}|w(z)|=\left|w\left(z_{0}\right)\right|=1 .
$$

Further, by Lemma 2, a number $m \geq 1$ exists with

$$
z_{0} w^{\prime}\left(z_{0}\right)=m w\left(z_{0}\right)
$$

In addition, we also suppose that

$$
w\left(z_{0}\right)=e^{i \theta} \quad(\theta \in[-\pi, \pi]) .
$$

Then, we have

$$
\begin{aligned}
\left|\frac{h\left(z_{0}\right)-1}{L-M h\left(z_{0}\right)}\right|= & \left|\frac{\alpha p\left(1+\sinh ^{-1}\left(w\left(z_{0}\right)\right)\right) \sqrt{\left(w\left(z_{0}\right)\right)^{2}+1}+\alpha z w^{\prime}\left(z_{0}\right)}{(L-M) p\left(1+\sinh ^{-1}\left(w\left(z_{0}\right)\right)\right) \sqrt{\left(w\left(z_{0}\right)\right)^{2}+1}-M Y_{4}(p, \alpha, z)}\right| \\
& \geq \frac{|\alpha| m-|\alpha| p\left(1+\sinh ^{-1}\left(\left|e^{i \theta}\right|\right)\right) \sqrt{e^{2 i \theta}+1}}{(L-M) p\left(1+\sinh ^{-1}\left(\left|e^{i \theta}\right|\right)\right) \sqrt{e^{2 i \theta}+1}+|M| Y_{5}(p, \alpha, e)} \\
& \geq \frac{|\alpha|\left(m-2^{\frac{1}{2}} p\left(1+\sinh ^{-1}(1)\right)\right)}{2^{\frac{1}{2}} p(L-M)\left(1+\sinh ^{-1}(1)\right)+|\alpha||M|\left[m+2^{\frac{1}{2}} p\left(1+\sinh ^{-1}(1)\right)\right]^{\prime}},
\end{aligned}
$$


where

$$
\mathrm{Y}_{4}(p, \alpha, z)=\left(\alpha p\left(1+\sinh ^{-1}\left(w\left(z_{0}\right)\right)\right) \sqrt{\left(w\left(z_{0}\right)\right)^{2}+1}+\alpha z w^{\prime}\left(z_{0}\right)\right)
$$

and

$$
\mathrm{Y}_{5}(p, \alpha, e)=\left(p|\alpha|\left(1+\sinh ^{-1}\left(\left|e^{i \theta}\right|\right)\right) \sqrt{e^{2 i \theta}+1}+|\alpha| m\right)
$$

Now, let

$$
k(m)=\frac{|\alpha|\left(m-2^{\frac{1}{2}} p\left(1+\sinh ^{-1}(1)\right)\right)}{2^{\frac{1}{2}} p(L-M)\left(1+\sinh ^{-1}(1)\right)+|\alpha||M|\left[m+2^{\frac{1}{2}} p\left(1+\sinh ^{-1}(1)\right)\right]},
$$

then,

$$
\begin{aligned}
k^{\prime}(m) & =\frac{2^{\frac{1}{2}}|\alpha| p(L-M)\left(1+\sinh ^{-1}(1)\right)+|M||\alpha|^{2} 2^{\frac{3}{2}} p\left(1+\sinh ^{-1}(1)\right)}{\left(2^{\frac{1}{2}} p(L-M)\left(1+\sinh ^{-1}(1)\right)+|\alpha||M|\left[m+2^{\frac{1}{2}} p\left(1+\sinh ^{-1}(1)\right)\right]\right)^{2}} \\
& >0 .
\end{aligned}
$$

Clearly, $k(m)$ is an increasing function; so,

$$
\max k(m) \geq k(1) \quad(m \geq 1),
$$

and

$$
\geq \quad \frac{\left|\frac{h\left(z_{0}\right)-1}{L-M h\left(z_{0}\right)}\right|}{2^{\frac{1}{2}} p(L-M)\left(1+\sinh ^{-1}(1)\right)+|\alpha||M|\left[1+2^{\frac{1}{2}} p\left(1+\sinh ^{-1}(1)\right)\right]} .
$$

From (17), we have

$$
\left|\frac{h\left(z_{0}\right)-1}{L-M h\left(z_{0}\right)}\right| \geq 1
$$

which is contradictory to the fact that $h(z) \prec \frac{1+L z}{1+M z}$. Thus, $|w(z)|<1$, and so, we obtain our desired result.

If we take $g(z)=\frac{z^{p+1} f^{\prime}(z)}{p f(z)}$ in Theorem 3, we have the following Corollary.

Corollary 4. If $f$ is in the class $\mathcal{A}_{p}$, has the form (3), and satisfies the condition

$$
1+\frac{\alpha}{p}\left(p+1+\frac{z f^{\prime \prime}(z)}{f^{\prime}(z)}-\frac{z f^{\prime}(z)}{f(z)}\right) \prec \frac{1+L z}{1+M z},
$$

where the limitation on $\alpha$ is

$$
|\alpha| \geq \frac{2^{\frac{1}{2}} p(L-M)\left(1+\sinh ^{-1}(1)\right)}{1-|M|-2^{\frac{1}{2}} p\left(1+\sinh ^{-1}(1)\right)(1+|M|)},
$$

then, $f \in \mathcal{S}_{p}^{*}($ pet $)$.

Proof. The proof is straightforward so it is left for the reader. 
Theorem 4. Let $g \in A_{p}$ be of the form (3) and satisfy the condition

$$
1+\frac{\alpha}{p} \frac{z^{1+p} g^{\prime}(z)}{(g(z))^{2}} \prec \frac{1+L z}{1+M z}
$$

where the limitation on $\alpha$ is

$$
|\alpha| \geq \frac{2^{\frac{1}{2}} p(L-M)\left(1+\sinh ^{-1}(1)\right)^{2}}{1-|M|-2^{\frac{1}{2}} p\left(1+\sinh ^{-1}(1)\right)(1+|M|)}
$$

then,

$$
\frac{g(z)}{z^{p}} \prec 1+\sinh ^{-1}(z) .
$$

Proof. Let us define a function

$$
h(z)=1+\frac{\alpha}{p} \frac{z^{1+p} g^{\prime}(z)}{(g(z))^{2}}
$$

where $h(z)$ is analytic in $\mathbb{D}$ and $h(0)=1$. Further, consider

$$
\frac{g(z)}{z^{p}}=1+\sinh ^{-1}(w(z))
$$

To show our result, we are required to show that $|w(z)|<1$. Now,

$$
h(z)=1+\frac{\alpha}{1+\sinh ^{-1}(w(z))}+\frac{\alpha z w^{\prime}(z)}{p\left(1+\sinh ^{-1}(w(z))\right)^{2} \sqrt{(w(z))^{2}+1}}
$$

and

$$
\begin{aligned}
\left|\frac{h(z)-1}{L-M h(z)}\right|=\left|\frac{\frac{\alpha}{1+\sinh ^{-1}(w(z))}+\frac{\alpha z w^{\prime}(z)}{p\left(1+\sinh ^{-1}(w(z))\right)^{2} \sqrt{(w(z))^{2}+1}}}{L-M\left(1+\frac{\alpha}{1+\sinh ^{-1}(w(z))}+\frac{\alpha z w^{\prime}(z)}{p\left(1+\sinh ^{-1}(w(z))^{2} \sqrt{(w(z))^{2}+1}\right.}\right)}\right| \\
=\left|\frac{\alpha p\left(1+\sinh ^{-1}(w(z))\right) \sqrt{(w(z))^{2}+1}+\alpha z w^{\prime}(z)}{(L-M) p\left(1+\sinh ^{-1}(w(z))\right)^{2} \sqrt{(w(z))^{2}+1}-M Y_{6}(p, \alpha, z)}\right|,
\end{aligned}
$$

where

$$
\mathrm{Y}_{6}(p, \alpha, z)=\left(\alpha p\left(1+\sinh ^{-1}(w(z))\right) \sqrt{(w(z))^{2}+1}+\alpha z w^{\prime}(z)\right)
$$

and suppose there occurs a point $z_{0} \in \mathbb{D}$ such that

$$
\max _{|z| \leq\left|z_{0}\right|}|w(z)|=\left|w\left(z_{0}\right)\right|=1
$$

Additionally, by Lemma 2, a number $m \geq 1$ exists with

$$
z_{0} w^{\prime}\left(z_{0}\right)=m w\left(z_{0}\right)
$$

We also suppose that

$$
w\left(z_{0}\right)=e^{i \theta} \quad(\theta \in[-\pi, \pi]) .
$$


Then, we have

$$
\begin{aligned}
&\left|\frac{h\left(z_{0}\right)-1}{L-M h\left(z_{0}\right)}\right|=\left|\frac{\alpha p\left(1+\sinh ^{-1}\left(w\left(z_{0}\right)\right)\right) \sqrt{\left(w\left(z_{0}\right)\right)^{2}+1}+\alpha z w^{\prime}\left(z_{0}\right)}{(L-M) p\left(1+\sinh ^{-1}\left(w\left(z_{0}\right)\right)\right)^{2} \sqrt{\left(w\left(z_{0}\right)\right)^{2}+1}-M Y_{7}(p, \alpha, z)}\right| \\
& \\
& \geq \frac{|\alpha| m-|\alpha| p\left(1+\sinh ^{-1}\left(\left|e^{i \theta}\right|\right)\right) \sqrt{e^{2 i \theta}+1}}{(L-M) p\left(1+\sinh ^{-1}\left(\left|e^{i \theta}\right|\right)\right)^{2} \sqrt{e^{2 i \theta}+1}+|M| Y_{8}(p, \alpha, e)} \\
& \geq \frac{|\alpha|\left(m-2^{\frac{1}{2}} p\left(1+\sinh ^{-1}(1)\right)\right)}{2^{\frac{1}{2}} p(L-M)\left(1+\sinh ^{-1}(1)\right)^{2}+|\alpha||M|\left[m+2^{\frac{1}{2}} p\left(1+\sinh ^{-1}(1)\right)\right]}
\end{aligned}
$$

where

$$
Y_{7}(p, \alpha, z)=\left(\alpha p\left(1+\sinh ^{-1}\left(w\left(z_{0}\right)\right)\right) \sqrt{\left(w\left(z_{0}\right)\right)^{2}+1}+\alpha z w^{\prime}\left(z_{0}\right)\right)
$$

and

$$
\mathrm{Y}_{8}(p, \alpha, e)=\left(p|\alpha|\left(1+\sinh ^{-1}\left(\left|e^{i \theta}\right|\right)\right) \sqrt{e^{2 i \theta}+1}+|\alpha| m\right)
$$

Now, let

$$
k(m)=\frac{|\alpha|\left(m-2^{\frac{1}{2}} p\left(1+\sinh ^{-1}(1)\right)\right)}{2^{\frac{1}{2}} p(L-M)\left(1+\sinh ^{-1}(1)\right)^{2}+|\alpha||M|\left[m+2^{\frac{1}{2}} p\left(1+\sinh ^{-1}(1)\right)\right]},
$$

then,

$$
\begin{aligned}
k^{\prime}(m) & =\frac{2^{\frac{1}{2}}|\alpha| p(L-M)\left(1+\sinh ^{-1}(1)\right)^{2}+|M||\alpha|^{2} 2^{\frac{3}{2}} p\left(1+\sinh ^{-1}(1)\right)}{\left(2^{\frac{1}{2}} p(L-M)\left(1+\sinh ^{-1}(1)\right)^{2}+|\alpha||M|\left[m+2^{\frac{1}{2}} p\left(1+\sinh ^{-1}(1)\right)\right]\right)^{2}} \\
& >0 .
\end{aligned}
$$

Clearly, $k(m)$ is an increasing function; so, $\max k(m) \geq k(1)$ for $m \geq 1$, so

$$
\begin{aligned}
& \left|\frac{h\left(z_{0}\right)-1}{L-M h\left(z_{0}\right)}\right| \\
& \quad \geq \frac{|\alpha|\left(1-2^{\frac{1}{2}} p\left(1+\sinh ^{-1}(1)\right)\right)}{2^{\frac{1}{2}} p(L-M)\left(1+\sinh ^{-1}(1)\right)^{2}+|\alpha||M|\left[1+2^{\frac{1}{2}} p\left(1+\sinh ^{-1}(1)\right)\right]} .
\end{aligned}
$$

From (17), we have

$$
\left|\frac{h\left(z_{0}\right)-1}{L-M h\left(z_{0}\right)}\right| \geq 1
$$

which is contradictory to the fact that $h(z) \prec \frac{1+L z}{1+M z}$. Thus, $|w(z)|<1$, and so, we obtain our desired result.

Corollary 5. If the function $f$ is in the class $\mathcal{A}_{p}$, has the form (3), and satisfies the condition

$$
1+\frac{\alpha f(z)}{z^{2 p+1} f^{\prime}(z)}\left(p+1+\frac{z f^{\prime \prime}(z)}{f^{\prime}(z)}-\frac{z f^{\prime}(z)}{f(z)}\right) \prec \frac{1+L z}{1+M z},
$$


where the limitation on $\alpha$ is

$$
|\alpha| \geq \frac{2^{\frac{1}{2}} p(L-M)\left(1+\sinh ^{-1}(1)\right)^{2}}{1-|M|-2^{\frac{1}{2}} p\left(1+\sinh ^{-1}(1)\right)(1+|M|)},
$$

then, $f \in \mathcal{S}_{p}^{*}($ pet $)$.

Theorem 5. Let $g \in A_{p}$ be of the form (3) satisfy the condition

$$
1+\frac{\alpha}{p} \frac{z^{1+2 p} g^{\prime}(z)}{(g(z))^{3}} \prec \frac{1+L z}{1+M z}
$$

where the condition on $\alpha$ is

$$
|\alpha| \geq \frac{2^{\frac{1}{2}} p(L-M)\left(1+\sinh ^{-1}(1)\right)^{3}}{1-|M|-2^{\frac{1}{2}} p\left(1+\sinh ^{-1}(1)\right)(1+|M|)}
$$

then,

$$
\frac{g(z)}{z^{p}} \prec 1+\sinh ^{-1}(z) .
$$

Proof. Let us define a function

$$
h(z)=1+\frac{\alpha}{p} \frac{z^{1+p} g^{\prime}(z)}{(g(z))^{2}}
$$

where $h(z)$ is analytic in $\mathbb{D}$ and $h(0)=1$. Further, consider

$$
\frac{g(z)}{z^{p}}=1+\sinh ^{-1}(w(z)) .
$$

Now, to prove our result we will required to show that $|w(z)|<1$. Now,

$$
h(z)=1+\frac{\alpha}{1+\sinh ^{-1}(w(z))}+\frac{\alpha z w^{\prime}(z)}{p\left(1+\sinh ^{-1}(w(z))\right)^{2} \sqrt{(w(z))^{2}+1}},
$$

and

$$
\begin{aligned}
\left|\frac{h(z)-1}{L-M h(z)}\right|=\left|\frac{\frac{\alpha}{1+\sinh ^{-1}(w(z))}+\frac{\alpha z w^{\prime}(z)}{p\left(1+\sinh ^{-1}(w(z))\right)^{2} \sqrt{(w(z))^{2}+1}}}{L-M\left(1+\frac{\alpha}{1+\sinh ^{-1}(w(z))}+\frac{\alpha z w^{\prime}(z)}{p\left(1+\sinh ^{-1}(w(z))\right)^{2} \sqrt{(w(z))^{2}+1}}\right)}\right| \\
=\left|\frac{\alpha p\left(1+\sinh ^{-1}(w(z))\right) \sqrt{(w(z))^{2}+1}+\alpha z w^{\prime}(z)}{(L-M) p\left(1+\sinh ^{-1}(w(z))\right)^{2} \sqrt{(w(z))^{2}+1}-M Y_{9}(p, \alpha, z)}\right|,
\end{aligned}
$$

where

$$
\mathrm{Y}_{9}(p, \alpha, z)=\left(\alpha p\left(1+\sinh ^{-1}(w(z))\right) \sqrt{(w(z))^{2}+1}+\alpha z w^{\prime}(z)\right) .
$$

Suppose there occurs a point $z_{0} \in \mathbb{D}$ such that

$$
\max _{|z| \leq\left|z_{0}\right|}|w(z)|=\left|w\left(z_{0}\right)\right|=1 .
$$


By Lemma 2, a number $m \geq 1$ exists with $z_{0} w^{\prime}\left(z_{0}\right)=m w\left(z_{0}\right)$. In addition, we also suppose that $w\left(z_{0}\right)=e^{i \theta}$ for $\theta \in[-\pi, \pi]$. Then, we have

$$
\begin{aligned}
\left|\frac{h\left(z_{0}\right)-1}{L-M h\left(z_{0}\right)}\right|= & \left|\frac{\alpha p\left(1+\sinh ^{-1}\left(w\left(z_{0}\right)\right)\right) \sqrt{\left(w\left(z_{0}\right)\right)^{2}+1}+\alpha z w^{\prime}\left(z_{0}\right)}{(L-M) p\left(1+\sinh ^{-1}\left(w\left(z_{0}\right)\right)\right)^{2} \sqrt{\left(w\left(z_{0}\right)\right)^{2}+1}-M Y_{10}\left(p, \alpha, z_{0}\right)}\right| \\
& \geq \frac{|\alpha| m-|\alpha| p\left(1+\sinh ^{-1}\left(\left|e^{i \theta}\right|\right)\right) \sqrt{e^{2 i \theta}+1}}{(L-M) p\left(1+\sinh ^{-1}\left(\left|e^{i \theta}\right|\right)\right)^{2} \sqrt{e^{2 i \theta}+1}+|M| Y_{11}(p, \alpha, e)} \\
& \geq \frac{|\alpha|\left(m-2^{\frac{1}{2}} p\left(1+\sinh ^{-1}(1)\right)\right)}{2^{\frac{1}{2}} p(L-M)\left(1+\sinh ^{-1}(1)\right)^{2}+|\alpha||M|\left[m+2^{\frac{1}{2}} p\left(1+\sinh ^{-1}(1)\right)\right]}
\end{aligned}
$$

where

$$
\mathrm{Y}_{10}\left(p, \alpha, z_{0}\right)=\left(\alpha p\left(1+\sinh ^{-1}\left(w\left(z_{0}\right)\right)\right) \sqrt{\left(w\left(z_{0}\right)\right)^{2}+1}+\alpha z w^{\prime}\left(z_{0}\right)\right)
$$

and

$$
\mathrm{Y}_{11}(p, \alpha, e)=\left(p|\alpha|\left(1+\sinh ^{-1}\left(\left|e^{i \theta}\right|\right)\right) \sqrt{e^{2 i \theta}+1}+|\alpha| m\right)
$$

Now, let

$$
k(m)=\frac{|\alpha|\left(m-2^{\frac{1}{2}} p\left(1+\sinh ^{-1}(1)\right)\right)}{2^{\frac{1}{2}} p(L-M)\left(1+\sinh ^{-1}(1)\right)^{3}+|\alpha||M|\left[m+2^{\frac{1}{2}} p\left(1+\sinh ^{-1}(1)\right)\right]},
$$

then,

$$
\begin{aligned}
k^{\prime}(m) & =\frac{2^{\frac{1}{2}}|\alpha| p(L-M)\left(1+\sinh ^{-1}(1)\right)^{2}+|M||\alpha|^{2} 2^{\frac{3}{2}} p\left(1+\sinh ^{-1}(1)\right)}{\left(2^{\frac{1}{2}} p(L-M)\left(1+\sinh ^{-1}(1)\right)^{3}+|\alpha||M|\left[m+2^{\frac{1}{2}} p\left(1+\sinh ^{-1}(1)\right)\right]\right)^{2}} \\
& >0 .
\end{aligned}
$$

Clearly, $k(m)$ is an increasing function; so,

$$
\max k(m) \geq k(1) \quad(m \geq 1)
$$

and

$$
\left|\frac{h\left(z_{0}\right)-1}{L-M h\left(z_{0}\right)}\right| \geq \frac{|\alpha|\left(1-2^{\frac{1}{2}} p\left(1+\sinh ^{-1}(1)\right)\right)}{2^{\frac{1}{2}} p(L-M)\left(1+\sinh ^{-1}(1)\right)^{3}+|\alpha||M|\left[1+2^{\frac{1}{2}} p\left(1+\sinh ^{-1}(1)\right)\right]} .
$$

From (17), we have

$$
\left|\frac{h\left(z_{0}\right)-1}{L-M h\left(z_{0}\right)}\right| \geq 1
$$

which is contradictory to the fact that $h(z) \prec \frac{1+L z}{1+M z}$. Thus, $|w(z)|<1$, and so, we obtain our desired result.

\section{Conclusions}

In this article, a subclass of regular multivalent functions in petal-shape domain has been introduced. These functions are then characterized with the help of some useful 
properties such as Fekete-Szegö problems and consequences to some special cases are discussed. We also derived some differential subordination implementation results involving $1+\sinh ^{-1}(z)$. These results can be generalized if we consider some other regions such as Lemniscate of Bernoulli region, cardioid region, nephroid domain, etc., instead of the Janowski domain.

In concluding our present investigation, we draw the attention of the interested readers toward the prospect of studying the basic or quantum (or $q$-) generalizations of the results we have developed in this paper. This direction of research was indeed influenced and motivated by a recently published survey-cum-expository review article by Srivastava [18]. However, as already demonstrated by Srivastava (see [18], p. 340; ([28], Section 5, pp. 1511-1512)), the (p, $q)$-variations of the proposed $q$-results will lead trivially to inconsequential research, because the forced-in parameter $\mathfrak{p}$ is obviously redundant. Furthermore, in light of Srivastava's more recent expository article [28], the interested readers should be advised not to be misled to believe that the so-called $k$-Gamma function provides a "generalization" of the classical (Euler's) Gamma function. Similar remarks will apply also to all usages of the so-called $k$-Gamma function, including (for example) the so-called $(k, s)$-extensions of the Riemann-Liouville and other operators of fractional integral and fractional derivatives.

Author Contributions: All authors contributed equally to this manuscript and approved the final version. All authors have read and agreed to the published version of the manuscript.

Funding: This research was funded by the Foundation of Excellent Youth Teachers of Colleges and Universities of Henan Province under grant no. 2019GGJS195.

Conflicts of Interest: The authors declare that they have no conflict of interest.

\section{References}

1. Ma, W.C.; Minda, D. A unified treatment of some special classes of univalent functions. In Proceedings of the Conference on Complex Analysis, Tianjin, China, 19-23 June 1992; Li, Z., Ren, F., Yang, L., Zhang, S., Eds.; Conference Proceedings and Lecture Notes in Analysis, Volume I; International Press: Cambridge, MA, USA, 1994; pp. 157-169.

2. Janowski, W. Extremal problems for a family of functions with positive real part and for some related families. Ann. Pol. Mathemat. 1970, 23, 159-177. [CrossRef]

3. Sharma, K.; Jain, N.K.; Ravichandran, V. Starlike functions associated with a cardioid. Afr. Mat. 2016, 27, 923-939. [CrossRef]

4. Cho, N.E.; Kumar, V.; Kumar, S.S.; Ravichandran, V. Radius problems for starlike functions associated with the sine function. Bull. Iran. Math. Soc. 2019, 45, 213-232. [CrossRef]

5. Geol, P.; Kumar, S.S. Certain class of starlike functions associated with modified sigmoid function. Bull. Malays. Sci. Soc. 2020, 43, 957-991. [CrossRef]

6. Bano, K.; Raza, M. Starlike functions associated with cosine function. Bull. Iran. Math. Soc. 2020, 47, 1513-1532. [CrossRef]

7. Kumar, S.S.; Ravichandran, V. Subordinations for functions with positive real part. Complex Anal. Oper. Theory 2018, 12, 1-13. [CrossRef]

8. Kumar, S.S.; Ravichandran, V. A subclass of starlike functions associated with a rational function. Southeast Asian Bull. Math. 2016, 40, 199-212.

9. Sokół, J.; Stankiewicz, J. Radius of convexity of some subclasses of strongly starlike functions. Zesz. Nauk. Politech. 1996, 19, 101-105.

10. Ali, R.M.; Cho, N.E.; Ravichandran, V.; Kumar, S.S. Differential subordination for functions associated with the lemniscate of bernoulli. Taiwan J. Math. 2012, 16, 1017-1026. [CrossRef]

11. Kumar, S.S.; Kumar, V.; Ravichandran, V. Sufficient conditions for starlike functions associated with the lemniscate of bernoulli. J. Inequal. Appl. 2013, 2013, 176. [CrossRef]

12. Kumar, S.S.; Khan, M.G.; Ahmad, B.; Mashwani, W.K. A class of analytic functions associated with sine hyperbolic functions. arXiv 2020, arXiv:2011.04875v1.

13. Paprocki, E.; Sokół, J. The extremal problem in some subclass of strongly starlike functions. Zesz. Nauk. Politech. Rzesz. Math. 1996, 20, 89-94.

14. Ahmad, B.; Khan, M.G.; Frasin, B.A.; Aouf, M.K.; Abdeljawad, T.; Mashwani, W.K.; Arif, M. On $q$-analogue of meromorphic multivalent functions in lemniscate of bernoulli domain. AIMS Math. 2021, 6, 3037-3052. [CrossRef]

15. Sharma, K.; Ravichandran, V. Applications of subordination theory to starlike functions. Bull. Iran. Math. Soc. 2016, 42, 761-777.

16. Khan, B.; Liu, Z.-G.; Srivastava, H.M.; Khan, N.; Darus, M.; Tahir, M. A study of some families of multivalent $q$-starlike functions involving higher-order $q$-Derivatives. Mathematics 2020, 8, 1470. [CrossRef] 
17. Khan, B.; Liu, Z.-G.; Srivastava, H.M.; Khan, N.; Tahir, M. Applications of higher-order derivatives to subclasses of multivalent q-starlike functions. Maejo Int. J. Sci. Technol. 2021, 15, 61-72.

18. Srivastava, H.M. Operators of basic (or $q$-) calculus and fractional $q$-calculus and their applications in geometric function theory of complex analysis. Iran. J. Sci. Technol. Trans. A Sci. 2020, 44, 327-344. [CrossRef]

19. Rehman, M.S.U.; Ahmad, Q.Z.; Srivastava, H.M.; Khan, N.; Darus, M.; Khan, B. Applications of higher-order $q$-derivatives to the subclass of $q$-starlike functions associated with the Janowski functions. AIMS Math. 2021, 6, 1110-1125. [CrossRef]

20. Khan, B.; Srivastava, H.M.; Tahir, M.; Darus, M.; Ahmad, Q.Z.; Khan, N. Applications of a certain integral operator to the subclasses of analytic and bi-univalent functions. AIMS Math. 2021, 6, 1024-1039. [CrossRef]

21. Khan, B.; Srivastava, H.M.; Khan, N.; Darus, M.; Ahmad, Q.Z.; Tahir, M. Applications of certain conic domains to a subclass of $q$-starlike functions associated with the Janowski functions. Symmetry 2021, 13, 574. [CrossRef]

22. Khan, B.; Srivastava, H.M.; Khan, N.; Darus, M.; Tahir, M.; Ahmad, Q.Z. Coefficient estimates for a subclass of analytic functions associated with a certain leaf-like domain. Mathematics 2020, 8, 1334. [CrossRef]

23. Srivastava, H.M.; Khan, B.; Khan, N.; Tahir, M.; Ahmad, S.; Khan, N. Upper bound of the third Hankel determinant for a subclass of $q$-starlike functions associated with the $q$-exponential function. Bull. Sci. Math. 2021, 167, 102942. [CrossRef]

24. Hu, Q.; Srivastava, H.; Ahmad, B.; Khan, N.; Khan, M.; Mashwani, W.; Khan, B. A subclass of multivalent Janowski type $q$-Starlike functions and its consequences. Symmetry 2021, 13, 1275. [CrossRef]

25. Kumar, S.S.; Arora, K. Starlike functions associated with a petal shaped domain. arXiv 2020, arXiv:2010.10072.

26. Keogh, F.R.; Merkes, E.P. A coefficients inequality for certain classes of analytic functions. Proc. Am. Math. Soc. 1969, 20, 8-12. [CrossRef]

27. Jack, I.S. Functions starlike and convex of order alpha. J. Lond. Math. Soc. 1971, 2, 469-474. [CrossRef]

28. Srivastava, H.M. Some parametric and argument variations of the operators of fractional calculus and related special functions and integral transformatioons. J. Nonlinear Convex Anal. 2021, 22, 1501-1520. 